\title{
Drug-Induced Lupus
}

Exposure to certain drugs has been associated with the development of lupus-like syndrome and this condition is termed drug-induced lupus or drug-related lupus. Patients with drug-induced lupus frequently show arthralgia or arthritis, rashes, pleurisy, pericarditis and fever. Pulmonary infiltrates can be seen. However, they do not often fulfill the revised criteria for systemic lupus erythematosus (SLE) by the American Rheumatism Association. The symptoms are usually mild, and severe multisystem involvement is rare. The disease clinically resembles idiopathic SLE in the elderly population in this aspect. Renal and CNS diseases are uncommon and antibodies against native DNA (double-stranded DNA) are not detected, which are the major differences between drug-induced lupus and idiopathic SLE. In contrast, antibodies against denatured DNA (single-stranded DNA) and histones are usually present in drug-induced lupus although their absence does not rule it out. Antinuclear antibodies (ANA) with homogeneous staining in immunofluorescence are observed in all cases of drug-induced lupus. Anti-Sm antibody is rarely detected and complement levels are generally within normal limits. Discontinuation of the drug results in disappearance of clinical symptoms in a few days to weeks, although ANA positivity remains for a certain period after withdrawal of the drug. However, steroids may also be necessary to treat certain conditions such as massive effusions in pleural or pericardial cavities.

See also p 587.

The demographic features of drug-induced lupus also differ from SLE. The male to female ratio is 1:9 in SLE whereas only a slight predominance in females is seen in drug-induced lupus. In addition, drug-induced lupus is more commonly found in the elderly population which reflects the higher incidence of underlying diseases such as ischemic heart diseases to be treated with implicated drugs. It is therefore often difficult to differentiate drug-induced lupus from idiopathic SLE in elderly individuals. Children receiving anticonvulsants can also be affected.

Drugs that induce lupus-like syndrome are divided into three categories: 1) drugs that frequently induce lupus-like syndrome, such as hydralazine, procainamide, 2) drugs that induce ANA without clinical manifestations, like procainamide, isoniazid, chlorpromazine, and 3) drugs that exacerbate SLE, such as sulfonamides and oral contraceptives (1). Anticonvulsants such as hydantoins and trimethadione are also implicated in druginduced lupus. Ethosuximide has been used to control both typical and atypical absence seizures. It is less toxic than trimethadione especially in terms of nephrotoxicity, but leukopenia and lupus-like syndrome are occasionally observed (2). Takeda et al (3) reported in this issue a case of lupus-like syndrome with renal involvement induced by ethosuximide. Renal biopsy revealed mild mesangial cell proliferation with multiple cellular crescents and arteriolar lesions. Removal of ethosuximide resulted in the disappearance of clinical symptoms and normalization of laboratory data including positive ANA and abnormal urinary sediments. It has been generally thought that renal involvement is not frequently encountered in drug-induced lupus. Renal biopsy findings are usually minimal. However, in rare cases, the histopathologic findings are indistinguishable from those of idiopathic SLE; various histopathologies including membranous proliferative and necrotizing glomerulonephritis have been documented in the literature $(4,5)$.

The pathogenesis of drug-induced lupus remains to be clarified. A number of possible mechanisms have been postulated; i.e. molecular mimicry, alteration of nucleic acids, abnormal immunoregulation under the influence of genetic background (6). Genetic factors include acetylator phenotype and histocompatibility antigens. Slow acetylators receiving procainamide or hydralazine develop lupus-like syndrome more rapidly than fast acetylators. Association of HLA-DR4 with hydralazine-induced lupus has been reported. Furthermore, certain chromosomal abnormalities may also be responsible as seen in this issue's case. Although genetic predisposition is obvious, it has not yet been made clear how the genetic background influences the susceptibility of an individual to develop drug-induced lupus. Unfortunately no animal models of drug-induced lupus have been developed. Further study will be necessary to clarify its pathogenesis but drug-induced lupus can be an experiment of nature to stress the importance of environmental factors in the induction of idiopathic SLE.

Nobuyuki MryasAKA, MD The First Department of Internal Medicine, School of Medicine Tokyo Medical and Dental University, 1-5-45, Yushima, Bunkyo-ku, Tokyo 113

\section{References}

1) Schur PH. Systemic lupus erythematosus. in: Rheumatology and Immunology, Cohen AS, Bennett JC, Eds. Grune \& Stratton, Orlando, FL, 1986, p. 247.

2) Livingston S, Rodriguez H, Greene CA, Pauli LL. Systemic lupus erythematosus. Occurrence in association with ethosuximide therapy. JAMA 203: 731, 1968.

3) Takeda S, Koizumi F, Takazakura E. Ethosuximide-induced lupus-like syndrome with renal involvement. Intern Med 35: 587, 1996. 
4) Sheikh TK, Charron RC, Katz A. Renal manifestations of drug-induced systemic lupus erythematosus. Am J Clin Pathol 75: 755, 1981.

5) Sturman SG, Kumararatne D, Beevers DG. Fatal hydralazine-induced systemic lupus erythematosus. Lancet 2: 1304, 1988 (letter).
6) Hess EV, Mongey AB. Drug-related lupus: the same as or different from idiopathic disease? in: Systemic Lupus Erythematosus, Lahita R, Ed. Churchill Livingston, New York, 1995, p.893. 